\title{
Os médicos de família das regiões de saúde de Lisboa e Vale do Tejo, Alentejo e Algarve vacinam-se contra a gripe sazonal? Porque sim ou porque não?
}

Ana Cebolais, ${ }^{*}$ Ana Costa, ${ }^{* *}$ Bruno Heleno, ${ }^{* * *}$ Marta Fonseca, ${ }^{* * * *}$ Rita Cunha Ferreira*

\section{RESUMO}

Objectivos: Determinar a proporção de vacinação contra a gripe nos médicos de família / clínicos gerais e caracterizar os motivos para a sua realização.

Tipo de estudo: Estudo observacional descritivo transversal.

Local: Centros de Saúde de Lisboa e Vale do Tejo, Alentejo e Algarve.

População: Médicos de família ou clínicos gerais exercendo actividade assistencial no último trimestre de 2008 , nas regiões de Lisboa e Vale do Tejo, Alentejo e Algarve.

Métodos: Amostra aleatória simples. Foi elaborado um questionário a partir da literatura sobre atitudes face à vacinação anti-gripal em profissionais de saúde, enviado por via postal. Calculou-se a estimativa intervalar, com nível de significância de 95\%, para a proporção de vacinação e para cada motivo.

Resultados: Dos 530 questionários enviados, obteve-se uma taxa de resposta de $42 \%$. Foram excluídos $28 \%$ dos questionários por preenchimento incompleto. Estimou-se uma proporção de vacinação de $62 \%$ (IC 95: 55-68\%). Os motivos que mais frequentemente justificaram a decisão de vacinação foram a protecção individual (100\%, IC95 96-100\%), a protecção do doente (83\% IC95 75-89\%), as recomendações internacionais e da Direcção-Geral da Saúde (81\%, IC95 74-89\%) e ter sido vacinado em anos anteriores (71\%, IC95 61-79\%). No grupo dos não vacinados, o motivo mais frequente foi o facto de a gripe ser uma doença benigna e autolimitada (81\%, IC95 49-89\%). Os restantes motivos foram menos apontados.

Conclusões: Encontrou-se uma proporção de vacinação superior à descrita na literatura. Os motivos mais importantes para a vacinação são concordantes com os encontrados na literatura, sendo a única excepção o peso atribuído às recomendações das sociedades científicas no sentido da vacinação. Nos médicos de família não vacinados, a convicção de que a gripe é uma doença benigna tem uma expressão maior que noutros estudos. A abordagem desta convicção poderá ser um ponto de partida para posteriores intervenções destinadas a aumentar a taxa de vacinação nesta população.

Palavras-Chave: Gripe, Humana; Vacina Contra a Gripe; Médico de Família; Atitudes dos Profissionais de Saúde.

\section{INTRODUÇÃO}

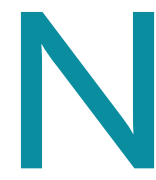

os nossos dias a gripe sazonal continua a ser uma causa importante de doença e de mortalidade. As crianças, idosos e doentes crónicos constituem os grupos de maior

*Médica Interna de Medicina Geral e Familiar, Centro de Saúde de Sete Rios **Médica Interna de Medicina Geral e Familiar, Centro de Saúde de Loures ***Médico Interno de Medicina Geral e Familiar, Centro de Saúde do Lumiar ****Médica Interna de Medicina Geral e Familiar, Centro de Saúde da Venda Nova risco, onde se observam as maiores taxas de morbilidade e/ou mortalidade. Nos EUA estima-se que, entre 1972 e 1992, a gripe tenha provocado cerca de 426.000 mortes. ${ }^{1}$ Ainda nos EUA, a gripe, em associação com a pneumonia, constitui a sétima causa de morte. Em Portugal, estima-se que, no período de 1990 a 1998, o número médio de mortes tenha sido de 1.773 por época gripal. ${ }^{2}$ A gripe sazonal tem também consequências socioeconómicas, sendo responsável por 10-12\% das cau- 
sas de absentismo laboral por doença nos EUA. ${ }^{3}$

Os profissionais de saúde têm características que os tornam importantes vectores na transmissão da gripe. ${ }^{4,5}$ Por um lado, contactam diariamente com doentes, com os seus familiares e com outros profissionais de saúde, tendo por isso uma exposição ao vírus influenza que é superior à da restante população. ${ }^{6}$ Num estudo de coorte realizado na Escócia estimou-se que até um quarto dos profissionais não vacinados venha a ter uma gripe sintomática, em cada época gripal. Admite-se que a conversão serológica assintomática possa ser ainda maior. ${ }^{7}$ Assim, os profissionais de saúde podem constituir um dos reservatórios do vírus influenza. Por outro lado, uma grande proporção dos profissionais de saúde com sintomas de gripe continua a trabalhar por pressão dos pares. ${ }^{8}$ Apesar de ser um comportamento bem intencionado, dá oportunidade à transmissão durante todo o período de contagiosidade da gripe, ou seja, desde o dia anterior ao início dos sintomas até cinco dias depois do final dos sintomas. A estas características acresce o facto de contactarem com uma população mais vulnerável à infecção e suas complicações. Foram, aliás, descritos vários surtos nosocomiais de gripe atribuídos a estes profissionais. ${ }^{9-12}$

Dois ensaios clínicos, realizados em unidades de cuidados prolongados geriátricos, mostraram que a vacinação dos profissionais de saúde conduziu a uma redução da mortalidade dos doentes internados (redução absoluta entre 7 e 8,8\%). ${ }^{13,14}$ Estes dois estudos são um dos principais argumentos presentes nas recomendações de várias organizações e sociedades científicas para a vacinação dos profissionais de saúde. ${ }^{15-19}$

Apesar das recomendações e de discussões éticas em torno deste tema, uma revisão sistemática internacional de artigos publicados entre 1990 e 2002 encontrou coberturas vacinais nos profissionais de saúde que oscilavam entre os $2,1 \%$ e os $82 \% .{ }^{5}$ Desde este período, admite-se que as coberturas vacinais têm vindo a aumentar. ${ }^{20,21}$ A previsão de uma pandemia de gripe poderá ter contribuído para este aumento. Num estudo realizado em médicos de família canadianos, 35,5\% foram vacinados contra a gripe. ${ }^{22}$ Em Portugal, na época gripal 2007-2008, os dados relativos a profissionais dos centros de saúde apontam para uma cobertura vacinal de $45 \%$ nos médicos, $54 \%$ nos enfermeiros e $50 \%$ nos outros grupos. $^{15}$
Na revisão sistemática supra referida ${ }^{5}$ é feito um levantamento das principais ideias que levam ou não à vacinação antigripal nos profissionais de saúde. Os factores que favorecem a decisão de se vacinar são: o desejo de protecção pessoal, o desejo de proteger os doentes, o acesso gratuito e conveniente à vacina, o facto de ter sido vacinado em anos anteriores e o exemplo dos pares. Pelo contrário, os factores que levam à decisão de não ser vacinado são: o receio de efeitos adversos, a convicção de que a vacina provoca gripe, sentir que se tem um risco reduzido de contrair a gripe, horários ou locais de administração da vacina pouco convenientes, convicção de que a gripe não é uma doença grave, convicção de que a vacina é ineficaz e o medo da picada. Na literatura internacional existem relatos de várias intervenções com o intuito de aumentar a taxa de cobertura da vacina antigripal nos profissionais de saúde. ${ }^{5}$ Existem duas características que estão presentes nas intervenções bem sucedidas: distribuição gratuita da vacina e intervenção educativa. Estas intervenções decorreram geralmente em três fases: informação (comunicação de mensagens chave por meios diversos como e-mail e panfleto), educação (sob a forma de sessões clínicas, conferências e vídeos) e vacinação (facilmente acessível, por exemplo através de carrinhos de vacinação que percorrem os vários serviços).

A investigação sobre a vacinação antigripal nos profissionais de saúde em Portugal tem escasseado mas poderá permitir identificar as áreas de intervenção mais adequadas para aumentar a cobertura vacinal.

Os médicos dos cuidados de saúde primários, ao contactarem diariamente com um elevado número de indivíduos doentes e saudáveis, possuem risco acrescido de infecção e transmissão da gripe. É ainda mais relevante em termos epidemiológicos se tivermos em consideração o frequente contacto com doentes com diversas comorbilidades e, portanto, com maior risco de complicações e consequente morbilidade e/ou mortalidade. ${ }^{2}$ Um estudo sugere que os médicos de família vacinados tendem a aconselhar a vacinação aos seus utentes idosos com maior frequência que os médicos não vacinados. ${ }^{22}$ Dada a importância da vacinação antigripal nestes profissionais de saúde, o aumento da sua vacinação é desejável.

Os objectivos deste trabalho são dar a conhecer a cobertura vacinal nos médicos de cuidados de saúde 
primários portugueses e os motivos que fundamentam a sua decisão de serem ou não vacinados contra a gripe sazonal. O conhecimento destes motivos poderá identificar áreas para posterior intervenção no âmbito da melhoria da taxa de vacinação nacional nos profissionais portugueses.

\section{MÉTODOS}

Foi realizado um estudo observacional transversal descritivo, por inquérito postal, aos médicos de família ou clínicos gerais a exercer funções assistenciais nos centros de saúde das regiões de saúde de Lisboa e Vale do Tejo, Alentejo e Algarve no último trimestre de 2008.

A maioria dos médicos dos cuidados de saúde primários em Portugal encontra-se ligada às administrações regionais de saúde (ARS). Assim, estas foram contactadas no sentido de serem fornecidas as listagens dos médicos de família e clínicos gerais a exercer funções assistenciais nos centros de saúde do território continental. Das cinco ARS contactadas tivemos acesso aos dados relativos à ARS Lisboa e Vale do Tejo, ARS Alentejo e ARS Algarve. Por este motivo, definiu-se que a nossa população acessível seriam os médicos de família e clínicos gerais a exercer funções assistenciais nestas regiões.

Para o cálculo da dimensão amostral assumimos que o estudo seria realizado numa população finita de 2215 médicos. Consideraram-se as prevalências de motivos relacionados com a decisão de vacinação próximas de $50 \%$ e que seria aceitável obter uma precisão absoluta dos resultados de 7,5\%. Desta forma chegou-se a uma dimensão amostral de 159 médicos. Admitindo uma taxa de resposta de $30 \%$, foi necessário enviar 530 questionários.

Para a amostragem utilizamos a listagem dos médicos de família / clínicos gerais que trabalharam em 2008, fornecidas pelas regiões de Lisboa e Vale do Tejo, Alentejo e Algarve. Foram excluídos os médicos internos e os médicos destacados para funções não assistenciais. Os nomes dos médicos foram numerados por ordem alfabética e utilizou-se um gerador de números aleatórios ${ }^{23}$ para seleccionar a amostra (amostra aleatória simples).

Para a construção do questionário foram elaboradas, pelos autores, perguntas a partir de motivos descritos na literatura. Estes motivos são apontados como eventuais justificações para a decisão de ser ou não vacinado contra a gripe. O questionário (Apêndice 1) consistiu num conjunto de perguntas fechadas e numa pergunta aberta («outros motivos») para os indivíduos vacinados e não vacinados. Foi testado num grupo de quinze médicos internos de Medicina Geral e Familiar. O objectivo do teste foi avaliar a legibilidade, detectar ambiguidades de linguagem ou de interpretação, identificar outros motivos que não estivessem presentes no questionário original e aferir o tempo de preenchimento. Após correcções, foi novamente testado num grupo de 15 médicos que cumpriam os critérios de elegibilidade.

No processo de colheita de informação foi garantida a confidencialidade dos dados: selecção aleatória dos participantes, recolha anónima dos questionários e utilização dos dados recolhidos apenas para o presente trabalho de estudo. O questionário foi enviado para os centros de saúde, endereçado aos médicos seleccionados, sendo acompanhado de dois envelopes: um envelope selado com o nome do médico e outro sem identificação. Solicitámos aos inquiridos que colocassem o questionário no envelope sem identificação e que, por sua vez, o introduzissem dentro do envelope identificado. Os envelopes identificados serviram para seleccionar os colegas que não necessitaram de rechamada. Os envelopes não identificados foram colocados num recipiente fechado de forma a manter o anonimato. Como estratégia de rechamada, após duas semanas foi enviada uma segunda carta aos médicos não respondentes.

A fase da recolha de dados decorreu durante os meses de Junho e Julho de 2009. Foi criada uma base de dados em SPSS versão $16 .{ }^{24}$ Foi aplicada estatística descritiva para tratamento de cada uma das variáveis. Para averiguar as diferenças entre respondentes e não respondentes no que diz respeito à idade e ao sexo, foram utilizados os testes de Mann-Whitney e Qui-quadrado, respectivamente. Para averiguar as diferenças entre os vacinados e não vacinados em relação à idade, ao sexo e às regiões de saúde onde exerceram a sua actividade profissional, foram também utilizados os testes de Mann-Whitney e Qui-quadrado. Calcularam-se intervalos de confiança de $95 \%$ para cada um dos motivos utilizando um método de Wald ajustado (OpenEpi versão 2.3)..$^{25}$ 


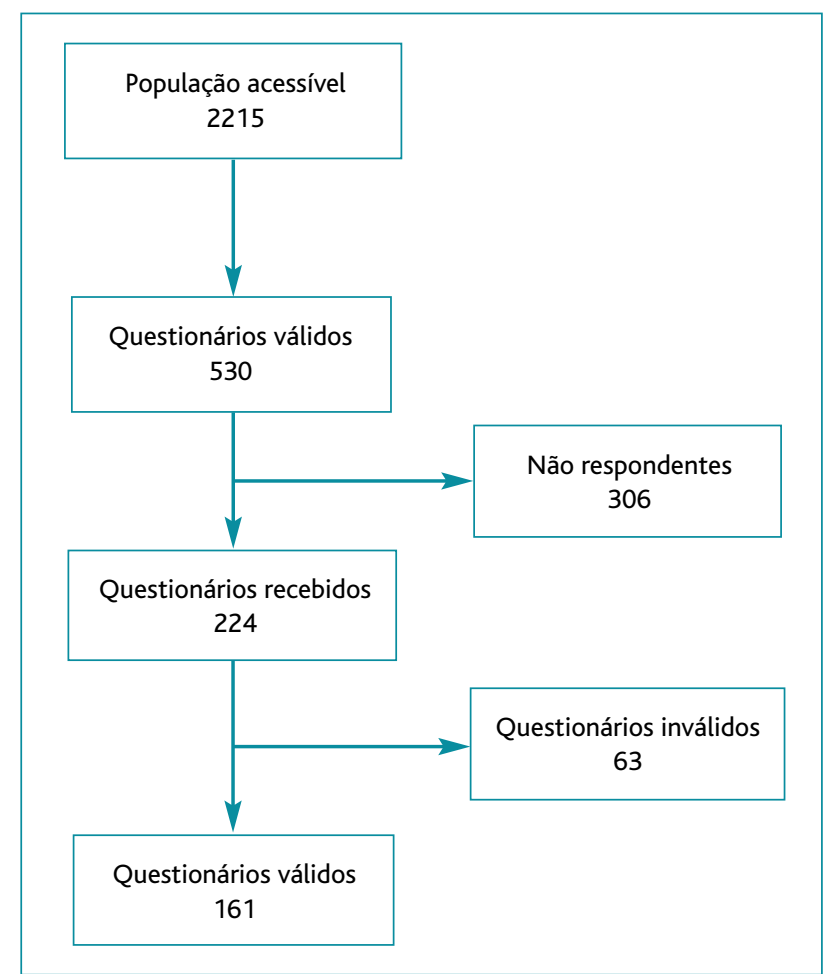

Figura 1. População acessível, questionários enviados, questionários recebidos e não respondidos, questionários válidos e inválidos.

\section{RESULTADOS}

Dos 530 questionários enviados, obtiveram-se 224 respostas (42\%), como se observa na Figura 1. Observou-se uma forte evidência estatística de que os respondentes eram ligeiramente mais velhos que os não respondentes (idade mediana 54 vs 53 anos, amplitude interquartil 52-57 vs 51-56 anos, $\mathrm{p}=0,001$ ). No entanto, não se encontraram diferenças quanto ao sexo (homens: $38,9 \%$ vs $39,7 \%, p=0,854)$ ou quanto à distribuição por ARS ( $\mathrm{p}=0,827)$.

A maioria dos respondentes, 139 (62\%, IC 95 55$68 \%$ ), foi vacinada contra a gripe sazonal na época de 2008-2009.

Comparando os respondentes vacinados com os não vacinados, não foram observadas diferenças com significado estatístico quanto à idade (idade mediana 53 anos e amplitude interquartil 50-55 em ambos os grupos, $\mathrm{p}=0,686$ ), quanto ao sexo (homens: $54,8 \%$ vs $65,4 \%$, $\mathrm{p}=0,119)$ ou quanto à distribuição por ARS $(\mathrm{p}=0,257)$.

Dos inquéritos recebidos, 63 (28\%) não foram preenchidos completamente, pelo que apenas se analisaram os motivos de 161 inquéritos (Figura 1). Entre inquéritos válidos e inválidos, não se encontraram diferenças de idade $(\mathrm{p}=0,304)$, sexo $(\mathrm{p}=0,193)$ ou quanto à distri-

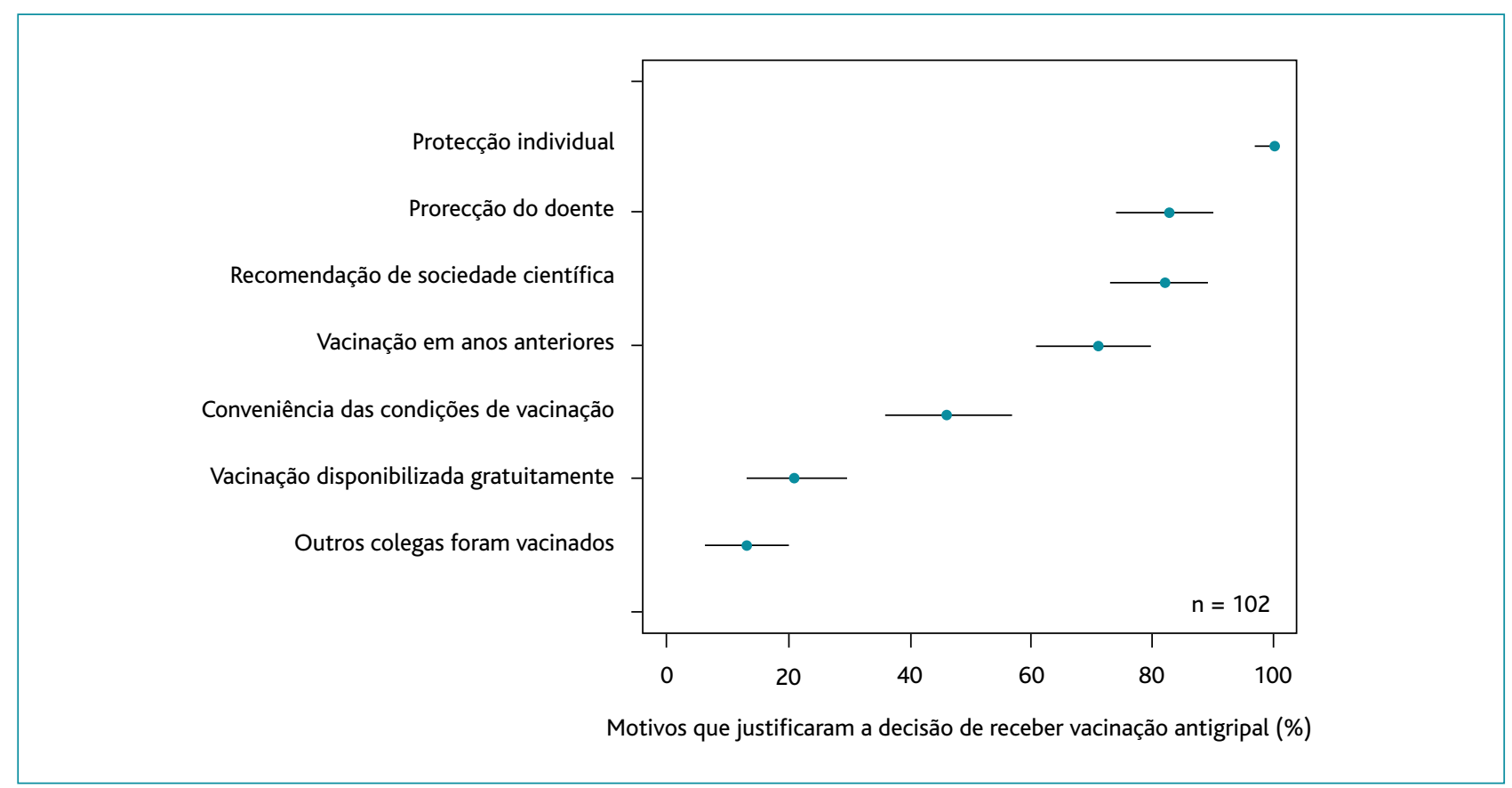

Figura 2. Motivos que justificaram a decisão de receber a vacinação antigripal. 
buição por ARS ( $\mathrm{p}=0,758$ ).

No grupo dos vacinados $(n=102)$, os motivos que mais frequentemente justificaram a decisão de vacinação (Figura 2) foram a protecção individual (100\%, IC95 96-100\%), a protecção do doente (83\% IC95 75-89\%), as recomendações internacionais e da Direcção-Geral da Saúde (81\%, IC95 74-89\%) e o ter sido vacinado em anos anteriores (71\% IC95 61-79\%).

No grupo dos não vacinados ( $\mathrm{n}=59$ ), o motivo mais frequentemente apontado (Figura 3 ) foi o facto de a gripe ser uma doença benigna e autolimitada (81\%, IC95 49-89\%). Alguns colegas justificaram a sua decisão por considerarem ter um baixo risco de contrair gripe $(41 \%$, IC95 29-53\%) e de transmitir gripe (30\%, IC95 20-43\%).

\section{DISCUSSÃO}

Este trabalho utilizou um questionário elaborado pelo grupo a partir dos principais motivos para vacinação ou não vacinação encontrados na literatura. $\mathrm{O}$ trabalho poderia ter beneficiado de um estudo qualitativo prévio, mas tal não foi possível, dados os escassos recur- sos e os custos elevados. Contudo, no questionário existia uma pergunta aberta que permitia ao inquirido acrescentar outros motivos. Para além disso, complementou-se o questionário discutindo-o com um perito na área da vacinação da Direcção-Geral da Saúde.

A garantia do anonimato e a realização de uma rechamada foram também preocupações da metodologia deste estudo, contribuindo para uma taxa de resposta (42\%) maior do que a esperada. Porém, alguns autores propõem o atingimento de uma taxa de resposta de $70 \%$ para inquéritos postais. ${ }^{26} \mathrm{Assim}$, poderá existir um viés de selecção, uma vez que os respondentes podem ter atitudes e convicções diferentes dos não respondentes. Admitimos que haja maior motivação dos vacinados em responder mas acreditamos que a caracterização qualitativa dos motivos em cada um dos grupos é menos vulnerável a este viés.

Apesar de se ter encontrado uma diferença estatisticamente significativa de idades entre respondentes e não respondentes, esta parece não ter qualquer relevância prática. Quanto às outras características demo-

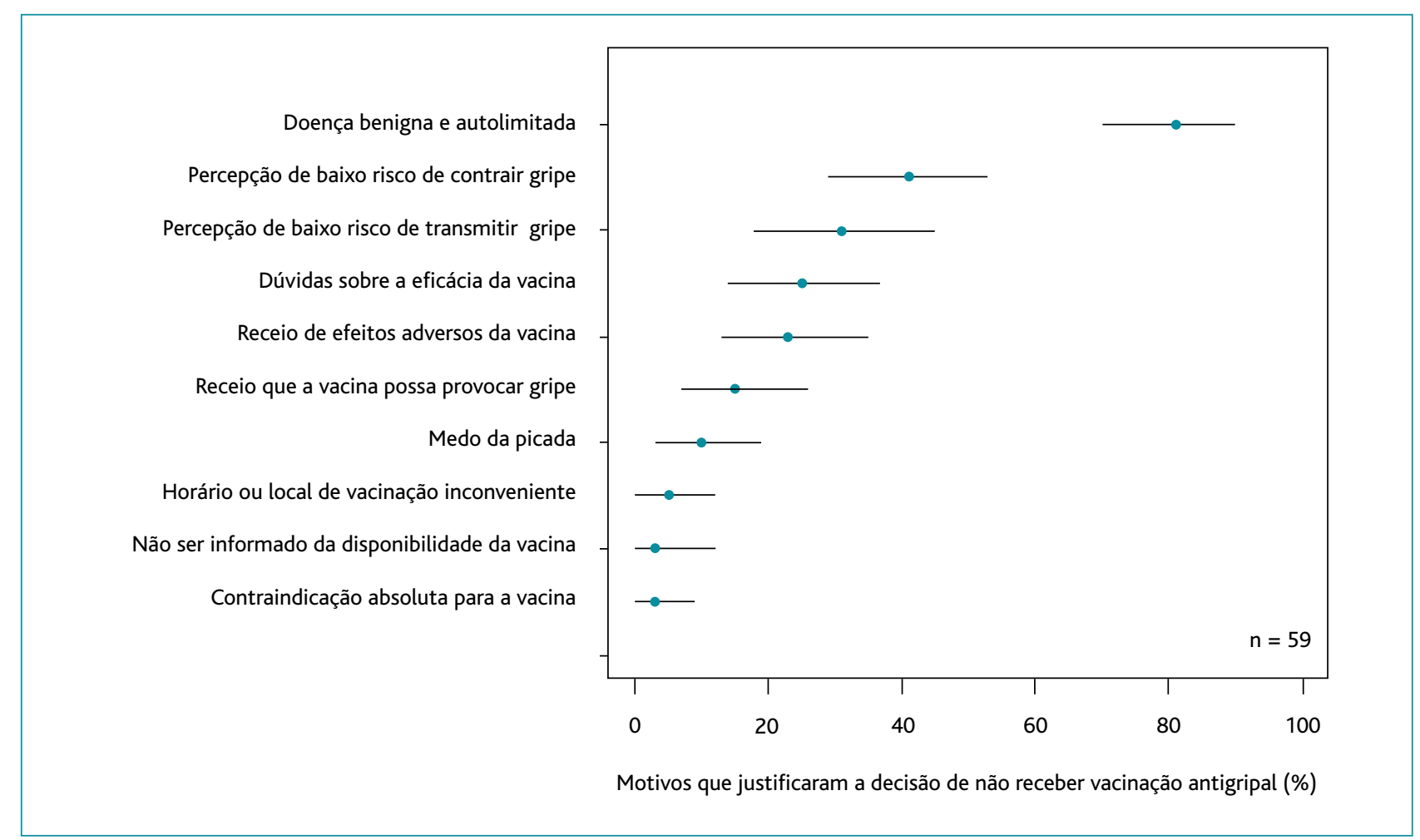

Figura 3. Motivos que justificaram a decisão de não receber vacinação antigripal. 
gráficas, não foram encontradas diferenças.

Mesmo com a realização de pré-teste, cerca de um terço dos questionários respondidos foram invalidados, facto que constituiu uma limitação do estudo. Como referido anteriormente, 63 questionários foram excluídos por preenchimento incompleto. Nestes questionários, os inquiridos apenas assinalaram respostas positivas. Havendo três hipóteses de resposta em cada pergunta, não seria lícito assumir que as questões não respondidas correspondiam a respostas negativas, sendo este o motivo que levou à sua exclusão. No entanto, não foram encontradas diferenças estatisticamente significativas entre as características dos questionários válidos e inválidos, pelo que consideramos que os resultados não terão sido substancialmente afectados.

Comparando os resultados com os encontrados na literatura nacional ${ }^{15} \mathrm{e}$ internacional ${ }^{20-22}$ obteve-se uma proporção de vacinação superior à descrita. A maioria dos estudos internacionais incluíam várias categorias de profissionais de saúde e foram realizados em contexto hospitalar. Apesar de este facto constituir uma explicação plausível para esta diferença, um estudo em médicos de família canadianos obteve uma proporção de vacinação inferior. ${ }^{22}$ Também os dados nacionais, que dizem respeito a médicos que trabalhavam em cuidados primários na época de 2007-2008, são inferiores aos resultados do nosso estudo. Pensamos, por isso, que a explicação mais razoável poderá estar relacionada com dois factores: a previsão de uma pandemia de gripe e também uma maior motivação das pessoas vacinadas em colaborar no estudo.

Quanto aos motivos mais importantes para os profissionais se vacinarem, encontra-se de forma geral concordância com a literatura. ${ }^{5}$ No entanto, no nosso estudo obteve-se uma maior proporção de médicos que atribuía a sua decisão às recomendações das sociedades científicas nacionais e internacionais. Uma possível explicação para este facto é a abrangência da Medicina Geral e Familiar, a qual poderá conduzir à necessidade dos médicos de família recorrerem com maior frequência às recomendações comparativamente a outros profissionais que foram alvo de estudos anteriores.

Nos médicos de família que optam por não se vacinar, a convicção de que a gripe é uma doença benigna e autolimitada tem uma maior expressão comparativamente com outros estudos. ${ }^{5}$ Talvez este resultado seja justificado parcialmente pelas diferenças entre a população do nosso estudo e a dos estudos citados, pois os médicos de família contactam com elevado número de casos de gripe, mas é nos cuidados de saúde secundários que se observa o maior número de complicações.

Apesar da distribuição gratuita e conveniente da vacina ser apontada como um factor crucial para aumentar as coberturas da vacinação antigripal nos profissionais de saúde ${ }^{5}$, é interessante notar que esse aspecto não foi valorizado pelos nossos inquiridos, quer vacinados, quer não vacinados.

Conclui-se com este trabalho que a proporção de vacinação antigripal na época de $2008-2009$ foi de $62 \%$ nos médicos de família / clínicos gerais das regiões de Lisboa e Vale do Tejo, Alentejo e Algarve. Os motivos que mais frequentemente justificaram a decisão de vacinação foram a protecção individual, recomendações internacionais e da Direcção-Geral da Saúde, protecção do doente e ter sido vacinado em anos anteriores. No grupo dos não vacinados, o motivo mais frequente foi o facto de a gripe ser uma doença benigna e autolimitada.

Neste trabalho existem duas importantes fontes de viés que podem condicionar a sua validade interna. São eles o viés de selecção resultante da baixa taxa de resposta e o viés de informação resultante do grande número de questionários incorrectamente preenchidos. Dentro dos objectivos deste trabalho, a caracterização da proporção de vacinados é mais vulnerável às fontes de viés que referimos. Todavia, este trabalho disponibiliza pela primeira vez resultados relativos às atitudes e convicções dos médicos de família do sul de Portugal face à vacinação antigripal. Poderão, portanto, ser tomados como ponto de partida por parte dos decisores de saúde.

Uma adequada campanha para aumentar a taxa de vacinação deve incluir três componentes: informação, educação e acessibilidade fácil e gratuita à vacina. ${ }^{5} \mathrm{Os}$ nossos resultados parecem sugerir que este último aspecto já terá sido atingido, uma vez que os nossos inquiridos não o identificaram como barreira à vacinação. Assim, pensamos que os decisores de saúde poderão investir na informação e educação. Para isso, será necessário produzir mais conhecimento.

Foi já demonstrada a redução na incidência de gri- 
pe nos médicos de família / clínicos gerais vacinados contra a gripe ${ }^{27}$. Contudo, falta demonstrar nos utentes a consequente redução da incidência de gripe clínica e de complicações graves. Só assim se podem esclarecer duas preocupações dos nossos inquiridos não vacinados: a eficácia da vacina e a percepção de baixo risco de transmitir gripe. Além disso, estudos posteriores poderão explorar o que é entendido como «doença benigna e autolimitada» já que é o motivo mais apontado pelos nossos inquiridos não vacinados.

No rescaldo da pandemia de gripe A, o presente trabalho deixa em aberto outras questões como saber se a proporção de vacinados e as atitudes e convicções se alteraram durante a época 2009-2010.

\section{REFERÊNCIAS BIBLIOGRAFICAS}

1. Simonsen L, Clarke MJ, Williamson GD, Stroup DF, Arden NH, Schonberger LB. The impact of influenza epidemics on mortality: introducing a severity index. Am J Public Health 1997 Dec; 87 (12): 1944-50.

2. Rascoa CL, Valente PM, Freitas MG. Enquadramento epidemiológico. In: Direcção-Geral da Saúde. Pandemia de gripe - plano de contigência nacional do sector da saúde para a pandemia de gripe. Lisboa: Direcção-Geral da Saúde; 2007. p.32.

3. Grandi P, Franco G. Practising evidence-based occupational health in workers' groups: how to prevent sickness absence caused by influenza. Occup Med (London) 2005 Jan; 55 (1): 7-9.

4. Meiklejohn G, Eickhoff TC, Graves, P I J. Antigenic drift and efficacy of influenza virus vaccines, 1976-1977. J Infect Dis 1978 Nov; 138 (5): 618-24.

5. Hofmann F, Ferracin C, Marsh G, Dumas R. Influenza vaccination of healthcare workers: a literature review of attitudes and beliefs. Infection 2006 Jun; 34 (3): 142-7.

6. Burls A, Jordan R, Barton P, Olowokure B, Wake B, Albon E, et al. Vaccinating healthcare workers against influenza to protect the vulnerable: is it a good use of healthcare resources? A systematic review of the evidence and an economic evaluation. Vaccine 2006 May 8; 24 (19): 4212-21.

7. Elder AG, O'Donnell B, McCruden EA, Symington IS, Carman WF. Incidence and recall of influenza in a cohort of Glasgow healthcare workers during the 1993-4 epidemic: results of serum testing and questionnaire. BMJ 1996 Nov 16; 313 (7067): 1241-2.

8. Weingarten S, Riedinger M, Bolton LB, Miles P, Ault M. Barriers to influenza vaccine acceptance: a survey of physicians and nurses. Am J Infect Control 1989 Aug; 17 (4): 202-7.

9. Horcajada JP, Pumarola T, Martínez JA, Tapias G, Bayas JM, de la Prada $M$, et al. A nosocomial outbreak of influenza during a period without influenza epidemic activity. Eur Respir J 2003 Feb; 21 (2): 303-7.

10. Salgado CD, Farr BM, Hall KK, Hayden FG. Influenza in the acute hospital setting. Lancet Infect Dis 2002 Mar; 2 (3): 145-55.

11. Slinger R, Dennis P. Nosocomial influenza at a Canadian pediatric hospital from 1995 to 1999: opportunities for prevention. Infect Control
Hosp Epidemiol 2002 Oct; 23 (10): 627-9

12. Health Protection Agency. Influenza A outbreak in a community hospital in south east Wales where few healthcare workers had received immunisation, February 2005. CDR Weekly 2005; 15(8): Disponível em: http://www.hpa.org.uk/cdr/archives/archive05/News/news0805.htm\# flu_A [acedido em 23/02/2010].

13. Carman WF, Elder AG, Wallace LA, McAulay K, Walter A, Murray GD, et al. Effects of influenza vaccination of health-care workers on mortality of elderly people in long-term care: a randomised controlled trial. Lancet 2000 Jan 8; 355 (9198): 93-7.

14. Potter J, Stott DJ, Roberts MA, Elder AG, O'Donnell B, Knight PV, et al. Influenza vaccination of health care workers in long-term-care hospitals reduces the mortality of elderly patients. J Infect Dis 1997 Jan; 175 (1): 1-6.

15. Direcção-Geral da Saúde. Circular Informativa 30/DSCS/DPCD. Lisboa: Direcção-Geral da Saúde; 2008.

16. World Health Organisation. Influenza vaccines. WHO Position Paper. Wkly Epidemiol Rec 2002 Jul 12; 77 (28): 230-9.

17. Van Essen GA, Palache AM, Forleo E, Fedson DS. Influenza vaccination in 2000: recommendations and vaccine use in 50 developed and rapidly developing countries. Vaccine 2003 May; 21 (16): 1780-5.

18. Orr P; National Advisory Committee on Immunization. An Advisory Committee Statement (ACS). National Advisory Committee on Immunization (NACI). Statement on influenza vaccination for the 20042005 season. Can Commun Dis Rep 2004 Jun 15; 30: 1-32.

19. Pearson ML, Bridges CB, Harper SA; Healthcare Infection Control Practices Advisory Committee (HICPAC); Advisory Committee on Immunization Practices (ACIP). Influenza vaccination of health-care personnel: recommendations of the Healthcare Infection Control Practices Advisory Committee (HICPAC) and the Advisory Committee on Immunization Practices (ACIP). MMWR Recomm Rep 2006 Feb 24; 55 (RR-2): $1-16$.

20. Harper SA, Fukuda K, Uyeki TM, Cox NJ, Bridges CB; Advisory Committee on Immunization Practices (ACIP), Centers for Disease Control and Prevention (CDC). Prevention and control of influenza: recommendations of the Advisory Committee on Immunization Practices (ACIP). MMWR Recomm Rep 2005 Jul 29; 54 (RR-8): 1-40.

21. Fiore AE, Shay DK, Broder K, Iskander JK, Uyeki TM, Mootrey G, et al.; Centers for Disease Control and Prevention (CDC); Advisory Committee on Immunization Practices (ACIP). Prevention and control of influenza: recommendations of the Advisory Committee on Immunization Practices (ACIP), 2008. MMWR Recomm Rep 2008 Aug 8; 57 (RR7): 1-60.

22. Baron G, De Wals P, Milord F. Vaccination practices of Quebec family physicians: influenza vaccination status and professional practices for influenza vaccination. Can Fam Physician 2001 Nov; 47: 2261-6.

23. Haahr M. RANDOM.ORG - Sequence Generator. Disponível em: http://www.random.org [acedido em 18/02/2008].

24. SPSS for Windows, Rel. 16.0.1. Chicago: SPSS Inc.; 2007.

25. OpenEpi version 2.3 - Open Source Epidemiologic Statistics for Public Health. Disponível em: http://www.openepi.com/Menu/OpenEpiMenu.htm [acedido em 26/05/2009].

26. Machin D, Campbell MJ. The design of studies for medical research. London. John Wiley and Sons; 2005. 
27. Michiels B, Philips B, Coenen S, Yane F, Steinhauser T, Stuyck S, et al. The effect of giving influenza vaccination to general practioners: a controlled trial. BMC Med 2006 Jul 10; 4: 17.

O autor Bruno Heleno é editor da Revista Portuguesa de Clínica Geral e declara não ter estado envolvido no processo de decisão editorial para este artigo.

\section{AGRADECIMENTOS}

Os autores gostariam de agradecer à Dra. Paula Valente, à Dra. Paula Broeiro, ao Dr. Paulo Nicola e ao Dr. Frederico do Rosário pelos seus comentários construtivos e sugestões, tanto ao protocolo do estudo, como ao manuscrito final do artigo.

\section{ENDEREÇO PARA CORRESPONDÊNCIA}

Ana Cebolais

Centro de Saúde de Sete Rios

Largo Professor Arnaldo Sampaio

1500-498 Lisboa

E-mail: anacebolais@hotmail.com

Recebido em 30/12/2009

Aceite para publicação em 29/03/2010

\section{ABSTRACT}

\section{DO FAMILY DOCTORS FROM LISBON AND TAGUS VALLEY, ALENTEJO AND ALGARVE HEALTH REGIONS IMMUNISE THEMSELVES AGAINST INFLUENZA? WHY?}

Objectives: Estimate the seasonal influenza immunisation rates of family doctors / general practitioners in 2008 and establish their reasons for getting or refusing immunization.

Study design: Cross-sectional study.

Setting: Primary care practices in South of Portugal (Lisbon and Tagus Valley, Alentejo and Algarve regions)

Participants: Family doctors / General Practitioners having regular, clinical contact with patients in the last trimester of 2008 in the aforementioned regions.

Methods: A questionnaire was developed, based on published studies on attitudes and motivations of healthcare workers towards influenza vaccines, and sent by mail to a random sample of family doctors / general practitioners. Both for immunisation rates and for each appointed reason $95 \%$ confidence intervals were calculated.

Results: There was a $42 \%$ response rate, although $28 \%$ were incompletely answered and excluded from analysis. Sixty two percent (Cl95 55-68\%) were immunized. In the immunized group, the most common reasons appointed were self-protection (100\%, Cl95\% 96-100\%), patient protection (83\% Cl95 75-89\%), guideline compliance (83\%, Cl95 75-89\%) and having a previous positive experience with the vaccine $(71 \%, \mathrm{Cl} 9561-79 \%)$. In the non-immunized group, the most frequent reason was considering influenza a benign and self-limited condition (81\%, Cl95 49-89\%). Other reasons were less often pointed out.

Conclusions: Compared with previous studies, higher immunization rates were found, although the relative importance of each reason was similar. Nevertheless, in the immunized group a higher number of participants appointed guideline compliance as a reason for being vaccinated and, in the non-immunized group, the number of participants that considered influenza a benign condition was higher than in previous studies. Addressing this conviction may be a starting point for interventions aimed to increase immunization rates in this population.

Keywords: Influenza, Human; Influenza Vaccines; Physicians, Family; Attitude of Health Personnel. 


\section{APÊNDICE 1 \\ QUESTIONÁRIO REALIZADO PELO GRUPO E ENVIADO POR VIA POSTAL À AMOSTRA DO ESTUDO}

\section{QUESTIONÁRIO}

Somos um grupo de médicos intemos do 3. ano de Medicina Geral e Familiar da região de Lisboa e gostariamos de contar com a sua participação num estudo de investigação. $O$ nosso objectivo é conhecer as motivações que levam os médicos de familia a realizar ou não a vacina da gripe.

Para colaborar connosco, pedimos-lhe apenas 1 minuto no sentido de responder a este curto questionário de forma anónima. Em seguida, deverá colocá-lo no envelope em branco e este, por sua vez, no interior do envelope RSF com o seu nome no remetente. Note que este processo não comprometerá o anonimato, permitindo no entanto o contacto posterior para «rechamada» dos não respondentes. Aguardamos uma resposta até 31 de Abril.

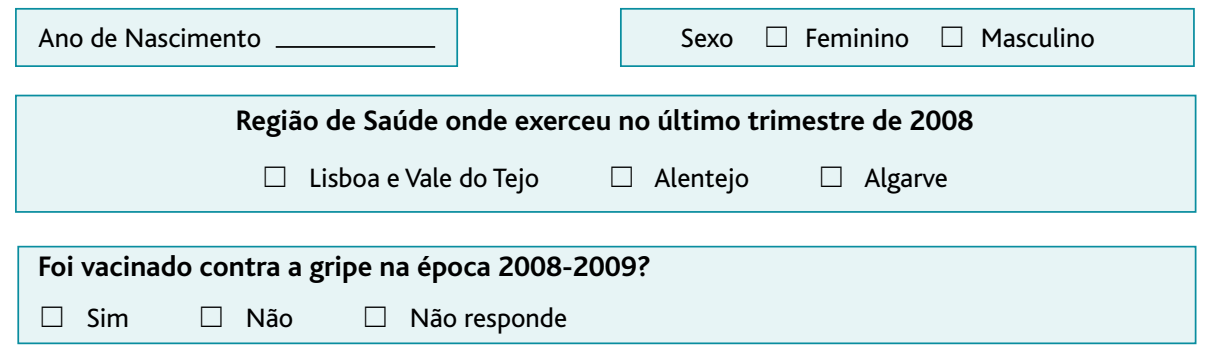

Consoante a resposta à pergunta anterior, responda apenas a uma das seguintes colunas:

\begin{tabular}{l}
\hline \multicolumn{3}{|c|}{ Se foi vacinado. Porquê? } \\
$\checkmark$ Porque a vacina me protege. \\
$\square$ Sim $\square$ Não $\square$ Não responde \\
$\checkmark$ Porque a vacina protege o doente. \\
$\square$ Sim $\square$ Não $\square$ Não responde \\
$\checkmark$ Porque fui vacinado em anos anteriores. \\
$\square$ Sim $\square$ Não $\square$ Não responde \\
$\checkmark$ Porque os meus colegas foram vacinados. \\
$\square$ Sim $\square$ Não $\square$ Não responde \\
$\checkmark$ Porque é uma recomendação internacional e da DGS. \\
$\square$ Sim $\square$ Não $\square$ Não responde \\
$\checkmark$ Porque a vacina foi gratuita. \\
$\square$ Sim $\square$ Não $\square$ Não responde \\
$\checkmark$ Porque as condições de vacinação me foram \\
convenientes. \\
$\square$ Sim $\square$ Não $\square$ Não responde \\
\end{tabular}

\begin{tabular}{|c|c|}
\hline 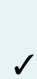 & $\begin{array}{l}\text { Se não foi vacinado. Porquê? } \\
\text { Porque a gripe é uma doenca benigna e autolimitada }\end{array}$ \\
\hline & Sim $\square$ Não $\square$ Não responde \\
\hline$\checkmark$ & Porque tenho dúvidas sobre a eficácia da vacina. \\
\hline & Sim $\square$ Não $\square$ Não responde \\
\hline & Porque a vacina pode provocar gripe. \\
\hline & Sim $\square$ Não $\square$ Não responde \\
\hline & Porque tenho receio dos efeitos adversos. \\
\hline & ] Sim $\square$ Não $\square$ Não responde \\
\hline & Porque tenho contra-indicação absoluta para a \\
\hline & Sim $\square$ Não $\square$ Não responde \\
\hline & Porque tenho baixo risco de contrair gripe. \\
\hline & ] Sim $\square$ Não $\square$ Não responde \\
\hline & Porque tenho baixo risco de transmitir gripe. \\
\hline & Sim $\square$ Não $\square$ Não responde \\
\hline & Porque tenho medo de picadas. \\
\hline & Sim $\square$ Não $\square$ Não responde \\
\hline 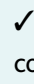 & $\begin{array}{l}\text { Porque o horário ou local para vacinação não foram } \\
\text { onvenientes. }\end{array}$ \\
\hline & ] Sim $\square$ Não $\square$ Não responde \\
\hline$V$ & Porque não fui informado acerca da disponibilidade. \\
\hline$\square$ & ] Sim $\square$ Não $\square$ Não responde \\
\hline
\end{tabular}

